Musées, Patrimoine et Culture scientifiques et techniques

$137 \mid 2011$

septembre - octobre 2011

\title{
Histoire locale et collections nationales : exposer pour mieux conserver
}

Local History and National Collections: exhibit to conserve better

Jean-Guy Michard

\section{(2) OpenEdition \\ Journals}

Édition électronique

URL : http://journals.openedition.org/ocim/958

DOI : 10.4000/ocim.958

ISSN : 2108-646X

Éditeur

OCIM

Édition imprimée

Date de publication : 1 septembre 2011

Pagination : 12-18

ISSN : 0994-1908

Référence électronique

Jean-Guy Michard, « Histoire locale et collections nationales : exposer pour mieux conserver », La

Lettre de I'OCIM [En ligne], 137 | 2011, mis en ligne le 01 septembre 2013, consulté le 20 avril 2019

URL : http://journals.openedition.org/ocim/958 ; DOI : 10.4000/ocim.958

Tous droits réservés 


\section{Histoire locale et collections nationales : exposer pour mieux conserver}

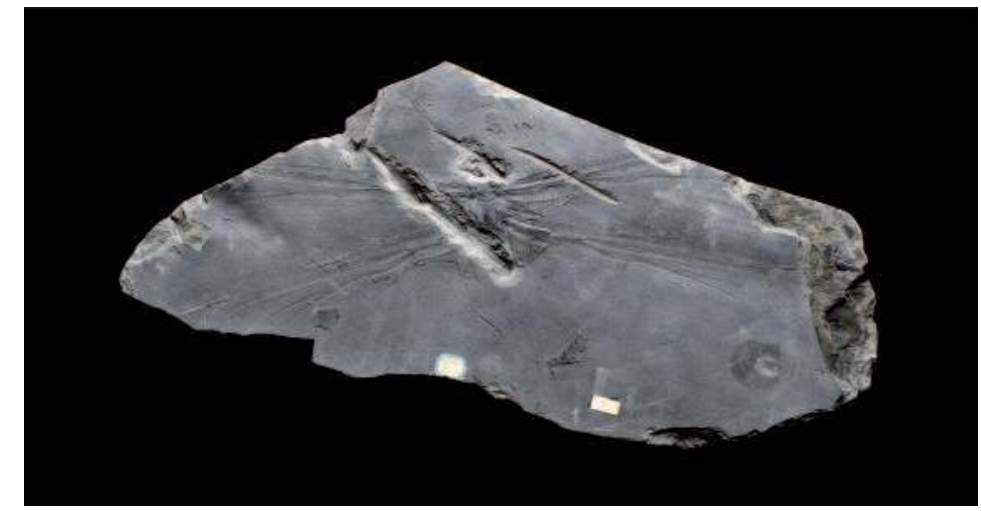

Le spécimen Meganeura monyi a été décrit et nommé par Charles Brongniart en 1885, peu de temps après sa découverte. Symbole de délicatesse, de vivacité et de liberté, cette libellule venue du fond des âges est I'emblème de la Ville de Commentry. (c) MNHN/M. Lemzaouda

* Jean-Guy Michard est maître de conférences au Muséum national d'Histoire naturelle, centre de recherche sur la conservation des collections, département des Galeries michard@mnhn.fr
Jean-Guy Michard *

Après avoir présenté les modalités de la conception et de la réalisation d'une exposition temporaire dans I'Allier en partenariat avec le Muséum national d'Histoire naturelle, l'auteur analyse la pertinence des nouvelles orientations prises par l'institution nationale envers les régions et $s$ interroge sur les limites de l'exercice en terme de conservation des collections.

\section{Historique du projet}

En août 2008, lors d'un déplacement professionnel dans l'Allier, nous avons souhaité savoir ce qu'il restait des célèbres mines de charbon de Commentry qui livrèrent de très nombreux fossiles de végétaux, de poissons et d'insectes. Plus d'un millier d'entre eux font partie des collections nationales conservées au Muséum et furent pour la plupart découverts entre 1880 et 1900 lorsque la mine, en grande partie à ciel ouvert, était dirigée par un ingénieur d'exception, Henri Fayol (1841-1925). Parmi ces fossiles se trouve un spécimen mondialement connu, Meganeura monyi, une libellule géante de $70 \mathrm{~cm}$ d'envergure. Nous sommes donc allés à l'Hôtel de Ville pour apprendre que les «tranchées » avaient toutes été remblayées ou transformées en étangs pour des raisons de sécurité. Cependant, la municipalité de Commentry avait adopté, il y a quelques années, cette libellule comme nouveau visuel pour la Ville, remplaçant ainsi la lampe et le pic de mineur devenus obsolètes (1). Le Maire de Commentry, Monsieur Gaby, avait demandé au Muséum national d’Histoire naturelle le prêt de ce fossile afin que les Commentryens puissent l'admirer, mais ses lettres étaient restées sans réponse, faute de destinataire précis. Aux questions qui 
nous furent posées concernant la possibilité de ce prêt, nous ne pûmes que conseiller au Maire d'adresser des demandes nominatives, l'une au professeur Michel Guiraud, directeur des collections du Muséum et l'autre au professeur Hervé Lelièvre, responsable des collections de Paléontologie, sans préjuger de leur réponse.

Cette réponse ne fut pas négative, bien que Meganeura soit un «type (2) » et un spécimen unique, mais assortie de conditions relatives, d'une part, à la sécurité du site et, d'autre part, aux modalités de transport et de mise en exposition. De plus, il ne pouvait être envisagé de ne prêter que ce seul spécimen afin de l'exposer dans quelque vitrine de l'Hôtel de Ville ; au contraire, Michel Guiraud souhaitait que fût organisée une véritable exposition, de taille modeste, certes, mais avec une réelle trame narrative scientifique sur la forêt du Carbonifère à l'emplacement actuel de Commentry et la formation du charbon ${ }^{(3)}$. Le rôle des mineurs, anonymes ou ingénieurs célèbres tels Stéphane Mony ou Henri Fayol, ainsi que celui de plusieurs savants du Muséum qui travaillèrent et publièrent sur ces fossiles et leur paléoenvironnement devaient aussi être présentés ${ }^{(4)}$.

\section{Une étude de faisabilité axée sur la sécurité}

La première démarche à entreprendre était donc de réaliser une étude de faisabilité portant sur le lieu de l'exposition et sa sécurisation. Pour cela les contacts plus étroits furent pris avec un certain nombre d'élus et de responsables

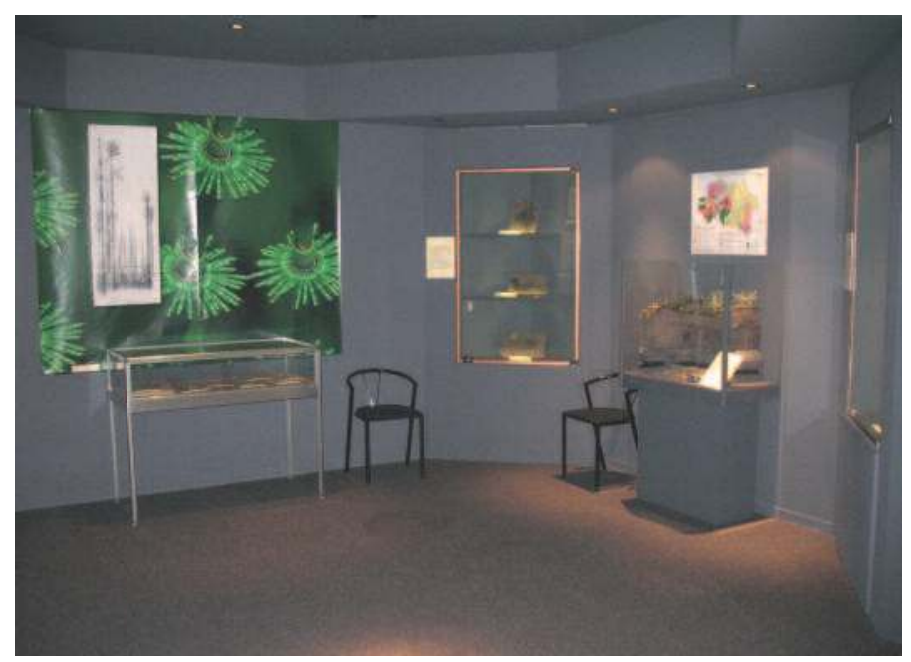

Les éclairages rasants en lumière froide par rubans de diodes électroluminescentes dans les vitrines ont remplacé avantageusement les néons plafonniers d'origine. Ils constituent une alternative aux fibres optiques lors de la rénovation de galeries permanentes. (c) J.G. Michard de la municipalité. La Ville de Commentry dispose, depuis 1993, d’une grande médiathèque, « La Pléiade », qui constitue un très bel espace culturel. Une salle scénographiée, " la salle du patrimoine », y est consacrée à Émile Mâle, enfant de Commentry, historien d'art et académicien, et communique avec une autre salle non meublée destinée à accueillir des expositions temporaires d'œuvres d'artistes locaux.

Lidée fut immédiatement de réunir ces deux espaces pour l'exposition, donnant ainsi un parcours contraint sans éclairage naturel dans la première partie et une possibilité de déambulation en ambiance plus lumineuse dans la seconde. L'ensemble représentait une surface d'environ $200 \mathrm{~m}^{2}$ une fois ajouté un large couloir de sortie ramenant les visiteurs vers le comptoir d'accueil. La salle scénographiée était, bien entendu, la plus sûre pour présenter iconographies et ouvrages originaux, objets et spécimens fossiles, ces derniers provenant principalement des collections du Muséum, mais pas uniquement. Trois points devaient cependant être améliorés : une sortie de secours donnant directement sur l'extérieur et non asservie à une détection incendie, donc facile à emprunter discrètement et rapidement ; des verres non feuilletés sur les vitrines ; certaines vitrines s'ouvrant du côté visiteurs. Une sécurité optimale des fossiles était le préambule à toute convention.

La question de la sortie de secours dans la partie sombre de l'exposition fut rapidement réglée par la commission de sécurité puisqu'une autre issue de secours proche de la précédente et facile à surveiller s'ouvre dans la seconde salle. Dès l'instant où les deux espaces étaient en communication permanente pendant l'ouverture au public, la condamnation d'une des issues n'était pas en contradiction avec les règlements de sécurité. En ce qui concerne la sécurité des vitrines, le vitrage de type « sécurit » existant fut renforcé par un film retardateur d'effraction, et huit petits détecteurs d'intrusion pour vitrines et leur centrale radio vinrent compléter le système d'alarme volumétrique existant. Il fut aussi prévu de poser des parcloses en bois peint autour de toutes les vitres, ce qui alliait un élément protecteur supplémentaire à un effet esthétique nouveau pour cet espace. Ces mesures eurent aussi pour effet de permettre une meilleure négociation de la prime d'assurance et constituent un bon exemple des nouvelles pratiques muséales en lien avec la valeur financière des collections d'Histoire naturelle (5).

Enfin, une ligne budgétaire importante par rapport au coût total prévisionnel de l'exposition fut allouée au conditionnement et aux transport des œuvres, afin de garantir au mieux l'intégrité de ces fossiles considérés, à juste titre, comme des objets fragiles. 


\section{Le choix des spécimens et ses conséquences inattendues}

L'objectif principal de l'exposition Trésors fossiles de Commentry était de créer un véritable événement régional en présentant sur le site de leur découverte, un nombre important de fossiles dont certains spécimens uniques d'une valeur scientifique inestimable. Le cœur de cette exposition était donc constitué par les fossiles du Carbonifère de Commentry et leur interprétation. Il ne s'agissait pas d'en exposer le plus grand nombre possible, mais de montrer la diversité des espèces représentées, notamment en ce qui concerne les végétaux. La sélection des spécimens fut particulièrement délicate, car nous savons tous que ce qui est très intéressant pour un scientifique est parfois totalement illisible pour un public non-spécialiste. Le sempiternel (faux) débat entre collections de recherche, collections d'exposition et éducation du visiteur ( "qu'il ne faut pas prendre pour un imbécile » (6)) fut évité grâce à une compréhension mutuelle des enjeux entre scientifiques et muséologues.

Une présélection des fossiles à présenter fut donc effectuée parmi le millier de spécimens conservés au Muséum national, tous taxons confondus ${ }^{(7)}$. Plus d'une centaine de pièces furent potentiellement retenues en concertation avec les chargés de conservation des collections pour arriver à une présentation finale de treize poissons, de quinze arthropodes dont une majorité d'insectes, dont le célèbre Meganeura monyi, et d'une soixantaine de restes botaniques allant de grand spécimens sur dalles de schiste à des ovules centimétriques de fougères. Mais le plus intéressant lors de cette sélection fut de repérer et de pouvoir isoler quelques spécimens qui présentaient des débuts d'altération dus à la dégradation de sulfures métalliques, généralement de la pyrite.

Or, un axe de recherche transversal se met actuellement en place au Muséum, concernant la conservation des fossiles et des minéraux dits " pyriteux », mobilisant des personnels de la direction des collections, du département des Sciences de la Terre et du centre de recherche sur la conservation des collections (CRCC), rattaché au département des Galeries. Des analyses chimiques poussées sont actuellement effectuées sur les produits de dégradation, des sulfates, afin de reconstituer des dynamiques de réactions et d'identifier les facteurs déclenchants. La constitution d'une base de données des gisements « à risques » s'avère indispensable étant donnée la variété des spécimens concernés. La préparation d'une exposition est donc un des moyens de contrôler l'état des collections, sachant qu' « une armée » de conservateurs ne pourrait le faire systématiquement et régulièrement pour les millions

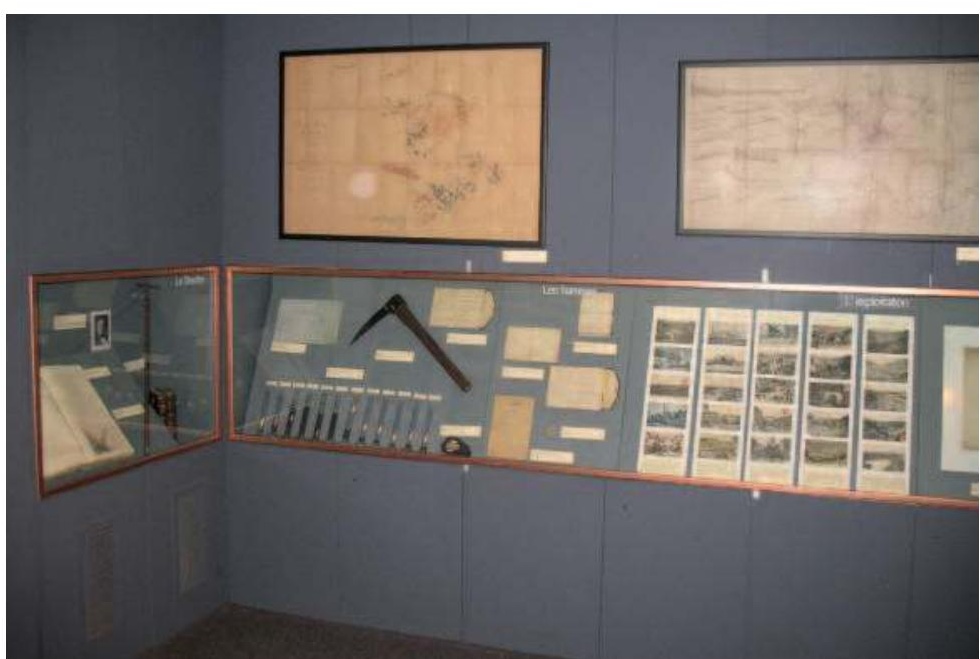

L'histoire de la mine de Commentry était illustrée par de nombreux prêts provenant de particuliers ou d'associations. (c) J.-G. Michard

d'échantillons composant les collections du Muséum. De plus, une telle opération permet de corriger ou d'attribuer des numéros d'inventaire tout en vérifiant la documentation scientifique et historique associée aux spécimens, ce qui est aussi nécessaire pour la rédaction des textes et des cartels de l'exposition.

\section{Un discours scientifique, une conception muséologique, un synopsis}

La phase de conception s'opéra selon les modalités classiques de rédaction d'un synopsis pour lequel les objectifs du projet furent définis collégialement. Les grandes lignes de la trame narrative furent assez rapidement validées grâce à la mise en place précoce d'un comité scientifique composé de représentants de la Ville de Commentry, du Muséum national d'Histoire naturelle et d'érudits locaux. Cette trame narrative avait pour but de permettre aux visiteurs de se déplacer dans le temps et dans l'espace, et surtout de constituer une manière agréable de découvrir la notion d'évolution de la vie sur Terre ainsi que les sciences qui permettent chaque jour de comprendre un peu mieux notre passé. Plusieurs thèmes furent abordés, allant du « particulier » au « général », en proposant un fil conducteur simple et clair, mais pas forcément linéaire à l'intérieur de chaque partie de l'exposition.

Mais au-delà de ce qui aurait pu être une simple présentation de fossiles, il nous a semblé important de replacer ces fossiles dans le contexte de leur formation, de leur mise au jour, et de leur étude. Cela constituait un deuxième objectif, certes ambitieux, mais indispensable pour faire comprendre aux visiteurs que ce patrimoine scientifique 
national résulte d'un enchaînement tout à fait particulier de processus et de conjonctures. La fossilisation de végétaux et d'animaux en milieu continental est déjà en soi un phénomène exceptionnel. Puis vient la lente transformation de ces dépôts organiques en charbon grâce à des conditions physico-chimiques spécifiques. Les mouvements de l'écorce terrestre vont ensuite participer à la mise en place de bassins houillers, que l'érosion rapprochera peu à peu de la surface. Il était dont important d'exposer quelques notions simples de géologie régionale avec une approche pédagogique, illustrée par des exemples concrets et des échantillons. Les différents niveaux de lecture devaient s'efforcer d'adopter un langage compréhensible par tous, ou de donner l'explication de certains termes incontournables.

Enfin, la trame narrative de cette exposition répondait à un troisième objectif. En passant d'un temps humain (l'exploitation de la mine et l'étude scientifique des fossiles, depuis environ 130 ans) à ces temps géologiques (la forêt du Carbonifère, il y a 300 millions d'années), nous avions l'ambition de faire découvrir un peu de l'histoire de la planète. La Terre n'est pas une boule rocheuse immuable, mais un système complexe en permanente évolution. Il y a 300 millions d'années, les hommes n'existaient pas, les dinosaures non plus, et les continents étaient très différents de ceux d'aujourd'hui. De nos jours, les dinosaures ont disparu ${ }^{(8)}$, mais les animaux et les végétaux continuent d'évoluer, les continents de se modifier et les hommes occupent une partie de la planète. Mais tout cela ne représente qu'un tout petit moment de l'histoire de la Terre, qui se forma il y a 4,5 milliards d'années, avec le reste du système solaire.

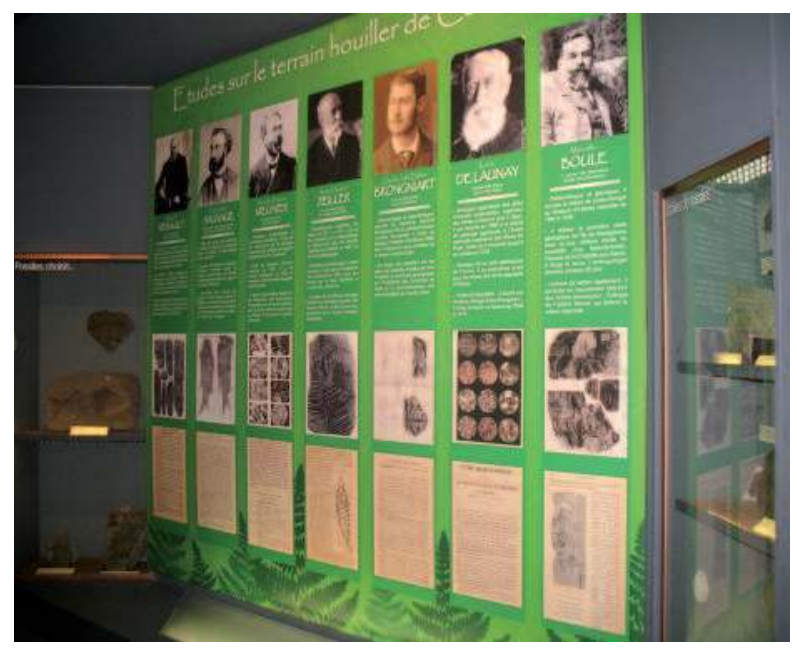

Le travail des savants était mis à I'honneur par une composition graphique de grande taille, encadrée par deux vitrines de spécimens dont certains font encore débat.

(c) J.-G. Michard
Le découpage de l'exposition et l'adaptation des différentes parties aux contraintes spatiales du lieu furent facilités par la rédaction du synopsis et le travail d'un plasticien-scénographe, Louis de Torhout, ayant une longue expérience des expositions à caractère scientifique. Les quatre premières thématiques, qui contenaient le plus d'objets, de documents et de spécimens géologiques et paléontologiques originaux, ont logiquement trouvé leur place dans la partie la plus sécurisée de l'exposition : l'introduction, la mine de Commentry et son histoire, l'action d'Henri Fayol, les fossiles et la géologie de Commentry. Les thématiques suivantes - les autres bassins houillers de la région, Commentry il y a 300 millions d'années, les représentations picturales de la forêt du Carbonifère et la frise des temps géologiques - trouvaient une bonne implantation dans la grande salle, plus lumineuse, permettant d'y présenter aussi un ensemble de plantes vivantes voisines de celles du Carbonifère.

\section{Les dispositifs particuliers}

Si le choix et la reconstitution d'une entrée de mine fidèle aux photographies d'époque ne posèrent que peu de problèmes grâce à l'efficacité des agents du centre technique municipal, la conception d'un dispositif de fin d'exposition fut beaucoup plus délicat. Évoquer le mode de transformation de la matière organique végétale en charbon relève de processus géologiques toujours délicats à donner à voir en exposition. Pour nous y aider, nous avions une maquette de cyclothème (9) provenant de l'ancienne Galerie de Paléobotanique à restaurer pour l'occasion, ainsi que le patient travail de vulgarisation audio-visuelle d'Hervé Lelièvre et de Pierre Debriette, qui fut géologue aux Charbonnages de France, spécialisé sur cette région.

Le souhait de présenter une image forte et synthétique à la fin de l'exposition, nous conduisit à décider la réalisation d'une maquette au 1/100 d'une forêt du Carbonifère. Ce dispositif alliait l'interprétation scientifique à l'imaginaire expographique et représentait un outil synoptique puissant permettant de donner une vision d'ensemble ${ }^{(10)}$. Outre son aspect ludique pour tous les publics, cette création, réalisée sous la direction de plusieurs scientifiques, notamment de Jean Broutin (11) permettait par sa taille (1,50 m x 1,50 m) et son échelle de rendre compte des différents éléments spécifiques à la formation du charbon : zones marécageuses et zones émergées, bord de lac et accumulation de végétaux morts, apports de matière et de sédiment par les rivières, notion de relief. La première contrainte était l'absence de végétaux du Carbonifère en modélisme. Fougères arborescentes, prêles géantes, Lycophytes et autres Sphénophytes durent être fabriqués un à un à partir de supports les plus inimaginables. Un deuxième souci était l'aspect statique de 


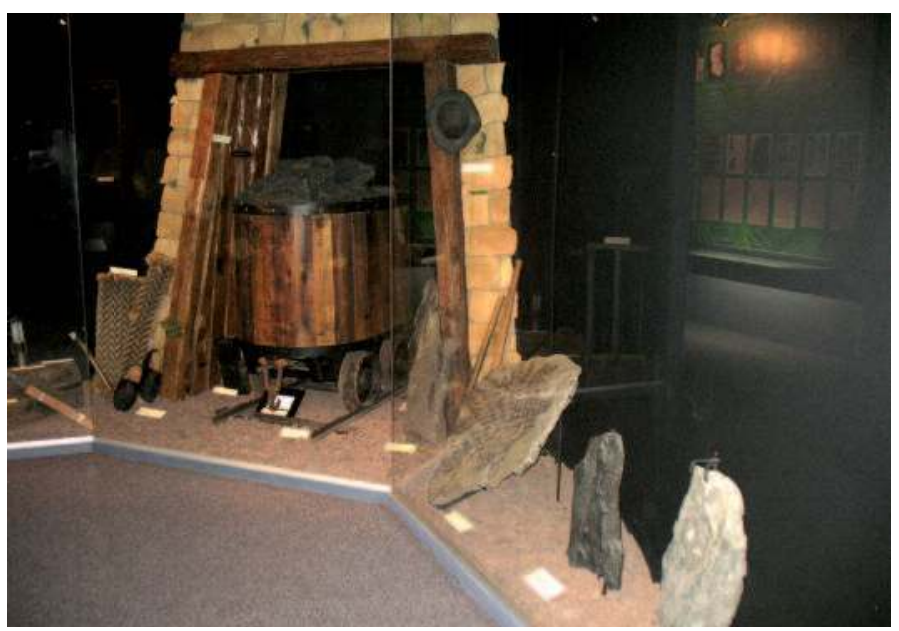

La mise en scène d'une entrée de galerie signée par Louis de Torhout a permis d'exposer un important patrimoine minier et de grands spécimens de la flore fossile.

(c) J.-G. Michard

cette maquette. Nous avons pu y remédier en intégrant sur sa large bordure un écran présentant une animation 3D de quelques minutes. Enfin, aucun référent de taille n'était présent sur ce modèle réduit de forêt, et les végétaux de $25 \mathrm{~m}$ de haut dans la réalité n'étaient guère impressionnants, bien que des schémas cotés et légendés agrémentaient aussi la bordure. L'idée de mettre un personnage au 1/100e ne pouvait qu'ancrer chez certains l'idée que les hommes vivaient à ces époques reculées. Nous choisîmes donc de forcer la dérision afin d'éviter tout amalgame : une voiture miniature actuelle fut placée dans un angle surélevé, sur une plaque évoquant l'asphalte annotée "échelle $1 / 100$ » . Différentes reproductions d'œuvres picturales représentant ce que pouvait être la forêt du Carbonifère complétaient cette immersion (12)

Enfin, la "frise du temps » fut réalisée de manière très conventionnelle à l'aide de quelques dizaines de mètres de corde ondulant sur les six mètres de long du couloir de sortie et portant une dizaine d'illustrations figurant les grandes étapes de l'évolution de la vie sur Terre. Mais l'inconvénient majeur de cette frise du temps était sa position en fin d'exposition, la seule possible du fait des contraintes architecturales. Une échelle des temps géologiques plus classique fut donc disposée sur le panneau d'introduction, à l'entrée, afin de permettre au visiteur de situer ces fossiles du Carbonifère dans le passé de notre planète.

\section{Une nouvelle politique d'ouverture du Muséum vers les régions}

Au delà de l'exercice délicat qui a consisté à fédérer pendant plus d'un an deux équipes, l'une travaillant à Commentry, l'autre à Paris, pour concevoir puis réaliser cette exposition, l'un des principaux paris gagnés fut sans doute la totale adhésion au projet de différentes structures en région. Le parisianisme parfois exacerbé de certains scientifiques du Muséum national d'Histoire naturelle par le passé a laissé des traces. Tout autant que l'image d'une certaine arrogance, justifiée ou non, du « Parisien » quel qu’il soit. En frôlant les 10000 entrées en cinq mois, l'exposition Trésors fossiles de Commentry a été un succès. Outre le travail des équipes de conception et des commissaires scientifiques, ce succès est aussi le résultat de la qualité des présentations, grâce à la participation active de particuliers de la région, à l'investissement de nombreux élus et personnels de la municipalité de Commentry, et la confiance que nous accordèrent plusieurs associations locales (et parisiennes), ainsi que celle du musée Anne de Beaujeu, à Moulins, et le muséum d'Histoire naturelle d'Autun. Il est évident que seule une attitude fondée sur l'écoute et le respect mutuel de la part de toute l'équipe parisienne permit d'aboutir à ce résultat. Nous retrouvons ici un phénomène comparable à celui des demandes de prêts aux musées en région pour les expositions temporaires de la Grande Galerie de l'Évolution à son ouverture. Très vite ce ne fut plus le prêt d'un objet qui fut demandé aux musées, mais des conseils sur l'illustration possible d'un thème ou d'un discours. Ne plus considérer les musées régionaux comme des magasins d'accessoires, on s'en doute, améliora sensiblement nos relations.

Le décret de 2001 définissant les nouveaux statuts du Muséum national d'Histoire naturelle a profondément modifié le fonctionnement de certaines structures, notamment celle de la direction des collections ${ }^{(13)}$. En se réorganisant transversalement au niveau de l'établissement, en se dotant d'organes de direction et de gestion, et en édictant un règlement des collections, cette direction statutaire a pu réinterpréter le sens de ses missions. Ce nouvel élan ainsi impulsé, l'implication de nombreux enseignants-chercheurs, et le soutien du directeur général et du directeur des collections ne sont pas étrangers à la réussite de ce projet en région. Ce fut aussi le cas, dans un autre registre de collaboration, pour le tout récent Paléospace l'Odyssée de Villers-sur-Mer. Accorder le prêt temporaire d'une centaine de spécimens fossiles provenant d'une collection dite "patrimoniale » à une structure non muséale accueillant du public en région, eût été probablement impossible il y a seulement une décennie. Cela ne reflète pas un laisser-aller complaisant, mais traduit au contraire une professionnalisation de la gestion et de la régie des collections.

Lors de l'étude de faisabilité, les conditions d'hygrométrie et de température avaient été contrôlées dans les vitrines destinées à accueillir les spécimens. Tout était donc prêt pour la mise en exposition... enfin, il ne restait quà déplacer les 
fossiles de 350 kilomètres. Des études avaient en effet démontré qu'une part importante des incidents affectant les collections avaient lieu lors des transports, même sur le site du Jardin des Plantes. Au fil de ces dernières années, les responsables scientifiques et techniques de la direction des collections, ont mis en place des procédures strictes de régie des œuvres, ainsi que des systèmes de conditionnement à base d'écrins de mousses neutres rigides ${ }^{(14)}$. La nature des mousses utilisées et le soin apporté à la confection de ces écrins garantissent non seulement une protection maximale lors des transports, mais aussi un meilleur rangement des spécimens à leur retour en réserve, quitte à ôter la plaque-couvercle pour voir le spécimen et diminuer le volume. Aucun bris ou fêlure ne furent à déplorer lors du transport, effectué selon les règles du convoyage, ni lors des manipulations sur le site de l'exposition uniquement autorisées aux paléontologues et au socleur. Cela fut attesté par les constats d'état effectués lors du conditionnement initial puis au retour des fossiles, et les photographies réalisées à cette occasion, font aujourd'hui partie de la documentation associée à chaque spécimen. Lorsqu'il existe des procédures adaptées mises au point par des professionnels de la conservation préventive d'une collection patrimoniale, la mise en exposition occasionnelle peut donc se révéler bénéfique : ce que nous confiait d'ailleurs, il y a une dizaine d'années, un conservateur venu installer un document ancien dans une exposition temporaire du Muséum national d'Histoire naturelle, après avoir éteint son luxmètre et ôté ses gants blancs.

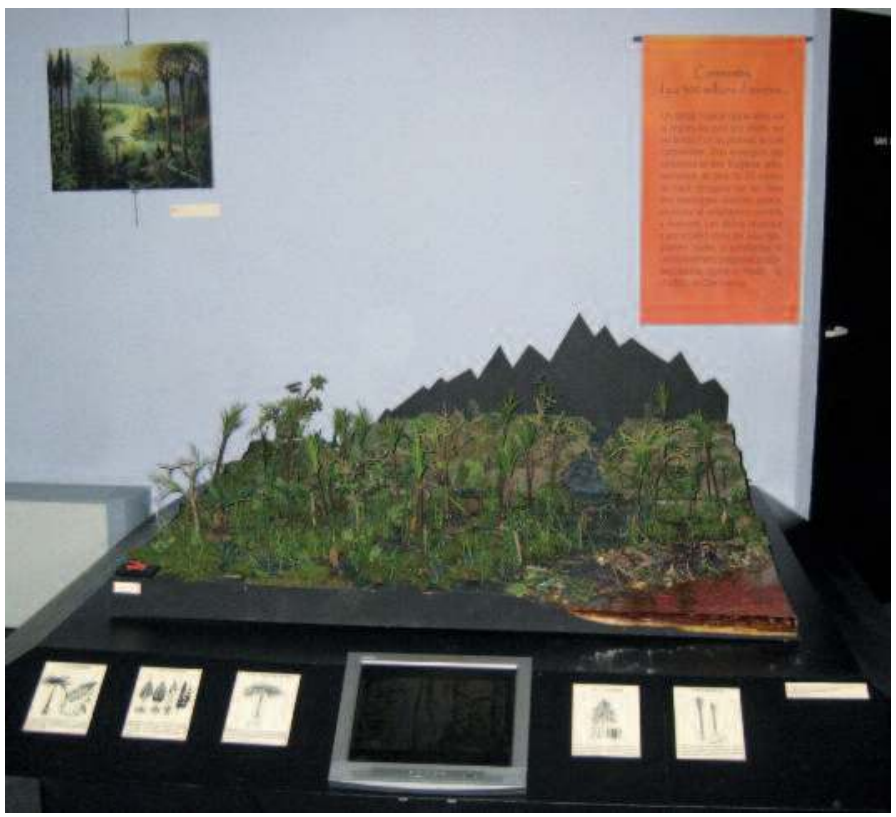

La maquette de la reconstitution de la forêt du Carbonifère a survécu à l'exposition : elle est actuellement présentée à la médiathèque de Commentry. (c) M. Champion

\section{Une expérience difficile à transposer}

Le Muséum national d'Histoire naturelle conserve environ 68 millions de spécimens. Ils proviennent de toute la planète, voire de la ceinture d'astéroïdes pour les météorites, et donc de nombreuses localités du territoire national. À l'exemple de cette aventure en terre bourbonnaise où le Muséum a tenu le rôle qui est le sien en accompagnant l'action culturelle et la diffusion de l'information scientifique en région, d'aucuns verrons une formidable opportunité d'accueillir, le temps d'une exposition, les trésors de leur patrimoine naturel. Hélas, trois fois hélas ! L'exercice n'est pas aussi simplement transposable. En premier lieu, nous l'avons vu, la structure d'accueil doit répondre à certaines contraintes de sécurité, tant pour les matériels exposés que pour l'accueil du public, et pouvoir offrir des conditions de conservation préventive satisfaisantes. Ensuite, s'il est théoriquement possible de faire voyager en toute sécurité (?) n’importe quel élément du patrimoine, certains demanderaient des mesures de préparation et de restauration si spécifiques et onéreuses qu'il ne saurait en être raisonnablement question. L'éléphant fossile (Mammuthus meridionalis) provenant de la localité de Durfort, dans le Gard, en est un exemple : du fait de la fragilité de ses os, une restauration et une consolidation complète seraient nécessaires en cas de désoclage. Enfin, la mission de diffusion du Muséum ne consiste pas simplement à exposer des objets d'Histoire naturelle, fussent-ils beaux, insolites ou historiques, et provenant des collections nationales. Ces derniers doivent illustrer un discours scientifique qui reflète l'activité de recherche toujours d'actualité fondée sur ce patrimoine.

Cette exposition a parfaitement répondu aux objectifs définis par ses concepteurs, et a donné lieu à une vérification de l'état des spécimens provenant de Commentry et des localités environnantes, ainsi qu’à leur documentation associée. La dimension historique et sociologique de cette présentation a aussi constitué un travail de mémoire permettant d'instaurer une réelle communication entre les générations et dont nous avons eu un témoignage direct ou par le biais du livre d'or. Quelques lignes tirées de la plaquette de l'exposition résument le désir d'ouverture du Muséum en région : "Souhaitons que l'expérience enrichissante qu'a constitué cette exposition des Trésors fossiles de Commentry soit la première d'une série qui nous mènera vers d'autres régions. Félicitons-nous d'avoir été parmi les animateurs de cette manifestation et cultivons cette notion du devoir de mémoire en l'enrichissant de celle des mémoires de la Terre» (15).

En plus de l'implication des personnes citées dans ce texte, cette entreprise n'aurait pu aboutir sans l'engagement opiniâtre d'Annie Lemoine, adjointe déléguée à la culture et au patrimoine, de Laurence Bourdier, directrice générale des 
services, d'Olivier Vignon, directeur du développement des actions locales, de Laurence Debowski, directrice de la médiathèque "la Pléiade ", pour la municipalité de Commentry, sans le savoir d'Alain Auclair, historien des industries et des techniques, ni sans l'amitié et le professionnalisme qui nous lient à nos collègues paléontologues Sylvain Charbonnier, Gaël Clément et Jean Dejax et à notre stagiaire de master, Mathias Champion, ainsi que l'aide précieuse de tous ceux qui participèrent à cette aventure, qu'ils soient de Commentry, de Paris ou d'ailleurs. La collaboration active des Archives nationales, de la Bibliothèque MinesParisTech, de l'association Gueules noires et Bocage, de la Société des Amis de Montluçon, de l'association Souvenir et Patrimoine du Mineur, et de la Société d'Encouragement pour l'Industrie nationale, furent aussi déterminantes. Que tous soient ici chaleureusement remerciés.

\section{Notes}

(1) Site officiel de la Ville de Commentry : www.ville-commentry.fr/

(2) Type : spécimen de référence attaché à un nom d'espèce.

(3) Carbonifère : système géologique caractérisé par de vastes dépôts de charbon produits par la putréfaction de végétaux dans des marécages (de -359,2 à -299 millions d'années)

(4) Fayol, H., de Launay, L., Meunier, S., Renault, B., Zeiller, R., Brongniart, C., Sauvage, E. et Boule, M. Études sur le terrain houiller de Commentry, Bulletin de la Société de l'Industrie minérale, Saint-Étienne, livraisons entre 1887 et 1890.

(5) La valeur financière des collections d'Histoire naturelle : confrontation des pratiques professionnelles. Rencontres professionnelles de l'OCIM, 25 janvier 2011 , Paris.

(6) De Wever, P., Guiraud, M., Cornée, A., Beckary, S. et Lasnier, B. Des collections en Sciences de la Terre pour quoi faire? Actes de la table ronde des 15 et 16 octobre 2002, Muséum national d'Histoire naturelle OCIM, 2005,166 p.
(7) Nom générique donné à toute unité systématique (espèce, genre, famille, ordre...).

(8) Les scientifiques s'accordent aujourd'hui à penser que certains dinosaures carnivores sont à l'origine du groupe des oiseaux. Ils n'ont donc pas vraiment disparu, telles que le montrent les récentes classifications phylogénétiques.

(9) Série de sédiments peu épais, lités et de composition différente, se succédant de manière régulière et liés à une sédimentation de caractère cyclique tels les sédiments houillers.

(10) Jacobi, D. La maquette en reconstitution savante et récit imaginaire dans les expositions d'archéologie, la Lettre de l'OCIM, n¹23, mai-juin 2009, pp. 15-23.

(11) Broutin, J. et Berthelin, M. Reconstitution d'un «paysage de l'époque houillère » revue à la lumière des découvertes récentes sur les cuticules végétales fossiles, Annales de Paléontologie, 92, 2006, pp. 91-104. De Wever, P., Broutin, J. et Néraudeau, D. Ces fossiles qui nous racontent la forêt, in Pays'âge, Musée d'Histoire naturelle de Lille, 2007, pp. 14-25.

(12) Champion, M. Évoquer un paysage disparu dans une exposition. Mémoire de Master 1 Hommes, Nature, Sociétés, spécialité Muséologie, Sciences, Cultures et Sociétés. Muséum national d'Histoire naturelle, Paris, 2010, $57 \mathrm{p}$

(13) Décret du 3 octobre 2001 publié le 7 octobre au Journal Officiel.

(14) Ces écrins sont constitués de plaques de mousse polyéthylène extrudée non réticulée de différentes épaisseurs au centre desquelles un logement à la forme du fossile est creusé, puis nappé de papier de soie neutre, le tout étant ensuite maintenu par des feuillards en plastiques. Les très grands spécimens sont en plus placés dans une caisse faite sur mesure.

(15) Collectif. Trésors fossiles de Commentry. Catalogue de l'exposition, co-production Adisséo - Muséum national d'Histoire naturelle et Ville de Commentry, 2010, 17 p. 